\title{
Direct observation of the nanoscale dynamics of membrane lipids in a living cell
}

\author{
Christian Eggeling ${ }^{1 *}$, Christian Ringemann ${ }^{1 *}$, Rebecca Medda ${ }^{1}$, Günter Schwarzmann ${ }^{2}$, Konrad Sandhoff ${ }^{2}$, \\ Svetlana Polyakova ${ }^{1}$, Vladimir N. Belov ${ }^{1}$, Birka Hein ${ }^{1}$, Claas von Middendorff ${ }^{1}$, Andreas Schönle ${ }^{1} \&$ Stefan W. Hell ${ }^{1}$
}

\begin{abstract}
Cholesterol-mediated lipid interactions are thought to have a functional role in many membrane-associated processes such as signalling events ${ }^{1-5}$. Although several experiments indicate their existence, lipid nanodomains ('rafts') remain controversial owing to the lack of suitable detection techniques in living cells ${ }^{4,6-9}$. The controversy is reflected in their putative size of 5-200 nm, spanning the range between the extent of a protein complex and the resolution limit of optical microscopy. Here we demonstrate the ability of stimulated emission depletion (STED) far-field fluorescence nanoscopy ${ }^{10}$ to detect single diffusing (lipid) molecules in nanosized areas in the plasma membrane of living cells. Tuning of the probed area to spot sizes $\sim 70$-fold below the diffraction barrier reveals that unlike phosphoglycerolipids, sphingolipids and glycosylphosphatidylinositol-anchored proteins are transiently $(\sim 10-20 \mathrm{~ms})$ trapped in cholesterol-mediated molecular complexes dwelling within $<20$-nm diameter areas. The noninvasive optical recording of molecular time traces and fluctuation data in tunable nanoscale domains is a powerful new approach to study the dynamics of biomolecules in living cells.
\end{abstract}

Unlike phosphoglycerolipids, sphingolipids and glycosylphosphatidylinositol (GPI)-anchored proteins are assumed to form molecular complexes or integrate, assisted by cholesterol, into $<200$-nm-sized lipid nanodomains impeding their diffusion in the plasma membrane $^{11}$. Featuring a diameter $d>200 \mathrm{~nm}$, the commonly used detection spot of a confocal microscope averages over such details of molecular diffusion (Fig. 1a). Electron microscopy provides the required resolution, but not in living cells ${ }^{12}$. Atomic force microscopy ${ }^{13}$, near-field optical microscopy ${ }^{14}$ and Förster resonance energy transfer ${ }^{15}$ could in principle provide an answer, but the direct visualization of lipid nanodomains rather than that of clustered proteins is challenged by the rapid diffusion of the lipids ${ }^{4}$. This rapid diffusion also prevents the fast tracking of single lipid molecules in their native cargo-free state $^{16-18}$. Although fluorescence recovery after photobleaching ${ }^{19,20}$ and confocal fluorescence correlation spectroscopy ${ }^{21-23}$ are able to map the diffusion, their resolution is diffraction-limited which requires, for example, mechanical contact with nanometric apertures ${ }^{24}$. In contrast, emergent stimulated emission depletion (STED) fluorescence microscopy ${ }^{10,25,26}$ (see Methods) opens up a new avenue to determine lipid diffusion, because its detection area can uniquely be downscaled in size by suppressing the fluorophore excitation at the outer parts of the focal spot (Fig. 1b).

Therefore we labelled sphingolipids, represented by sphingomyelin and ganglioside GM1, a GPI-anchor as well as the putatively 'nonintegrating' phosphoethanolamine with the fluorophore Atto647N and studied their dynamics in the plasma membrane of living cells (see Methods). Control experiments indicated that the dye label had no observable influence on the dynamic behaviour of the lipids or the GPI-anchor (Supplementary Fig. 8). An average of $<10$ labelled molecules per $\mu \mathrm{m}^{2}$ membrane area ensured the presence of single fluorescent molecules in the detection area. The measurements were performed in living mammalian cells under physiological conditions. Specifically, we targeted arbitrary points on the plasma membrane and recorded the fluorescence bursts of molecules crossing the focal spot. Figure 1c and e show single-molecule fluorescence time traces of phosphoethanolamine, measured using the regular confocal and the subdiffraction detection area downsized by STED to $d \approx 40 \mathrm{~nm}$, respectively. Besides demonstrating the ability of the STED microscope to detect single molecules in living cells, the traces show a uniform reduction of the burst length, accounting for the reduced transit time of a phosphoethanolamine molecule through the subdiffraction sized spot.

Next we recorded a similar pair of confocal and STED traces of sphingomyelin molecules (Fig. 1d and f). The confocal measurement does not show obvious differences between the sphingomyelin and phosphoethanolamine diffusion. Containing $\sim 1,000$ photons per molecule at an average transit time of $10 \mathrm{~ms}$, the fluorescence bursts are rather homogeneous in height and length for both lipids. In contrast, the STED microscope reveals a whole range of burst durations for sphingomyelin and hence a substantial difference to phosphoethanolamine. Whereas the phosphoethanolamine traces feature sharp peaks only, in the case of sphingomyelin the sharp peaks are accompanied by longer bursts. Thus the reduction of the detection area far below that provided by confocal microscopy reveals that the diffusion of sphingomyelin is strongly heterogeneous.

An important observation is that STED microscopy reduces the fluorescence burst length but hardly its peak height. The detected photon count-rate of a typical molecular trace peaks at $100 \mathrm{kHz}$, which enables the analysis of STED traces with a good signal-to-noise ratio. Hence we plotted a frequency histogram displaying the number of counts along with the burst duration for about 500 bursts recorded for phosphoethanolamine and sphingomyelin (Fig. 1g, h and Supplementary Fig. 2). For phosphoethanolamine we found just a single type of diffusion, characterized by burst durations $<1 \mathrm{~ms}$. Phosphoethanolamine shows no observable sign of heterogeneous diffusion in the plasma membrane. In contrast, the sphingomyelin histogram reveals a broad distribution of burst durations from short events that are similar to that of phosphoethanolamine, to longer events with burst durations ranging up to $>50 \mathrm{~ms}$.

To quantify the lipid dynamics further, we applied fluorescence correlation spectroscopy (FCS) averaging over many traces from individual molecules diffusing in and out of a well-known focal spot ${ }^{27}$. The decay of the correlation curve yields the average molecular transit time 


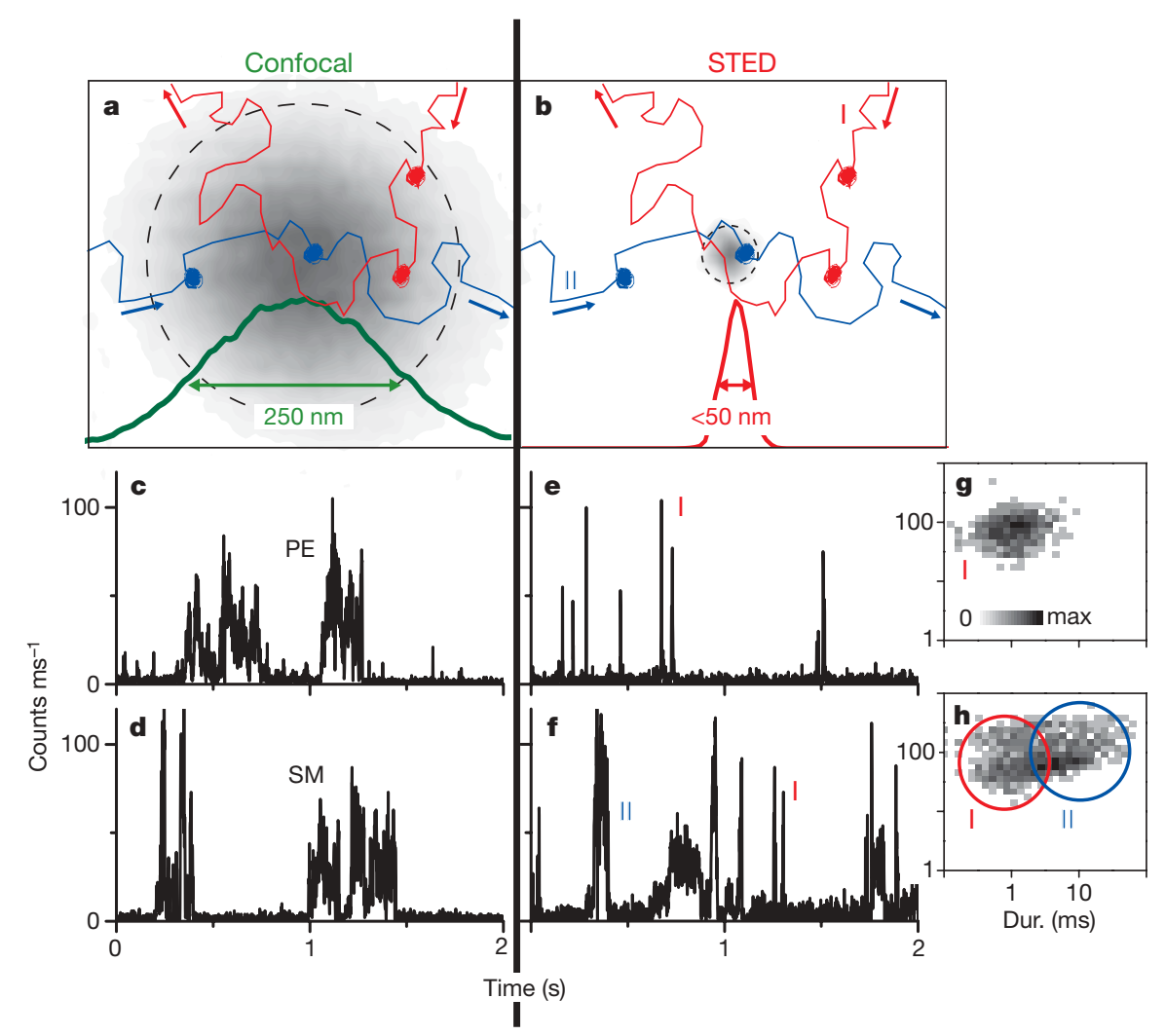

Figure 1 | STED microscopy time traces of single-molecule diffusion in live cell plasma membrane. a, b, Molecules may move freely and/or be transiently trapped on small spatial scales. The large detection area of a confocal microscope (a) cannot discern such details. However, the subdiffraction spot created by STED (b) is able to discriminate between lipids that diffuse freely (I) and those that are hindered (II) during their passage. c-h, Fluorescence bursts from single-diffusing Atto647N-labelled

through the focal area (Supplementary Information). The confocal correlation curves recorded for sphingomyelin are only slightly shifted compared to those recorded for phosphoethanolamine (Fig. 2a). Because both curves can be described by normal diffusion of a single species, we cannot infer whether the diffusion of sphingomyelin is inhomogeneous on small spatial scales, or just slightly slower than phosphoethanolamine. This is different in the correlation curves recorded for $d \approx 40 \mathrm{~nm}$ using STED, because here, marked disparities between phosphoethanolamine and sphingomyelin diffusion become apparent (Fig. 2b). Owing to the smaller detection area, the phosphoethanolamine curve is shifted towards shorter time scales, but still represents a single normal diffusion. In contrast, the sphingomyelin curve now features much longer time scales, and can be fitted only by a model assuming two dissimilar modalities of focal transits or, alternatively, anomalous diffusion of the lipids (Supplementary Information). To investigate whether the longer focal transit time of sphingomyelin is associated with cholesterol, we added cholesterol oxidase to deplete the cholesterol in the membrane. Figure $2 \mathrm{c}$ shows that the longer transit times disappear.

STED microscopy enabled us to scale the diameter of the focal detection area from $d=250 \mathrm{~nm}$ down to $30 \mathrm{~nm}$ after increasing the intensity of the STED light (Supplementary Fig. 1). The accompanying decrease in the transit time reveals the multifaceted dynamics of the membrane molecules ${ }^{23,24}$. The transit time for phosphoethanolamine decreases in proportion to the $\sim 70$-fold reduction in focal detection area (Fig. 3), indicating that phosphoethanolamine is indeed freely diffusing with a diffusion coefficient $D=(5 \pm 2) 10^{-9} \mathrm{~cm}^{2} \mathrm{~s}^{-1}$. This is different for sphingomyelin. For a relatively large subdiffraction spot diameter $d>160 \mathrm{~nm}$, the diffusion of sphingomyelin appears normal and just 1.5-2.5 times slower than that of phosphoethanolamine. phosphoethanolamine (PE) and sphingomyelin (SM) lipids detected with a confocal (c, d) and a STED spot $(\mathbf{e}, \mathbf{f})$, and a frequency plot of the value pairs of fluorescence counts per millisecond and duration (dur.) of selected bursts of the STED recording for phosphoethanolamine ( $\mathrm{g}, 497$ bursts) and sphingomyelin (h, 539 bursts). By ensuring that a freely diffusing molecule spends only little time in the detection area, STED microscopy distinguishes hindered (II) from free diffusion (I).

Likewise, for $80 \mathrm{~nm}<d<160 \mathrm{~nm}$, the spot size is not small enough to safely quantify anomalous diffusion of sphingomyelin. However, for $d<80 \mathrm{~nm}$, we can separate free from hindered diffusion and analyse the sphingomyelin correlation data by applying a model of two distinct modalities of focal transits: free and hindered diffusion. Considering that the free diffusion exhibits the same transit time $\tau_{D 1}$ as phosphoethanolamine (compare Fig. $1 \mathrm{~g}$ and $\mathrm{h}$ ), we calculated that a fraction of $A_{2} \approx 60 \%$ of sphingomyelin molecules crosses the $d=30$ $80 \mathrm{~nm}$ large areas within a transit time $\tau_{D 2}$ which is $>10$ times longer than $\tau_{D 1}$, reaching a plateau at $\sim 10 \mathrm{~ms}$.

This observation of a restricted minimum transit time through nanoscale areas rules out the possibility that sphingomyelin diffuses freely (but just more slowly) in the plasma membrane, because the hallmark of free diffusion is a transit time that scales with $d^{2}$; compare $\tau_{D 1}$. Therefore the hindered diffusion of sphingomyelin and the longer transit time $\tau_{D 2}$ must be due to a brief trapping of these molecules. The average trapping period $\tau_{\text {trap }} \sim 10 \mathrm{~ms}$ is calculated by subtracting $\tau_{D 1}$ from $\tau_{D 2}$. The fact that $\tau_{\text {trap }}$ is constant for $d<60 \mathrm{~nm}$ indicates a single trapping event within the probed areas. Similar trapping was observed for the Atto647N-labelled GPI-anchor (Fig. 3) and the ganglioside GM1 (Supplementary Fig. 6) with $D \approx 3$ and $5 \times 10^{-9} \mathrm{~cm}^{2} \mathrm{~s}^{-1}, \tau_{\text {trap }} \approx 18$ and $11 \mathrm{~ms}$, and $A_{2} \approx 35$ and $45 \%$, respectively.

Notably, the addition of the cholesterol-depleting agent cholesterol oxidase causes sphingomyelin, the GPI-anchor, and GM1 to exhibit a linear dependence of the transit time on $d^{2}$, which is indicative of the fact that the trapping has been reduced. Although cholesterol oxidase may also induce other changes in the membrane, it primarily depletes the cholesterol. Alternative cholesterol depletion by $\beta$-cyclodextrin also reduces the trapping (Supplementary Fig. 5). 


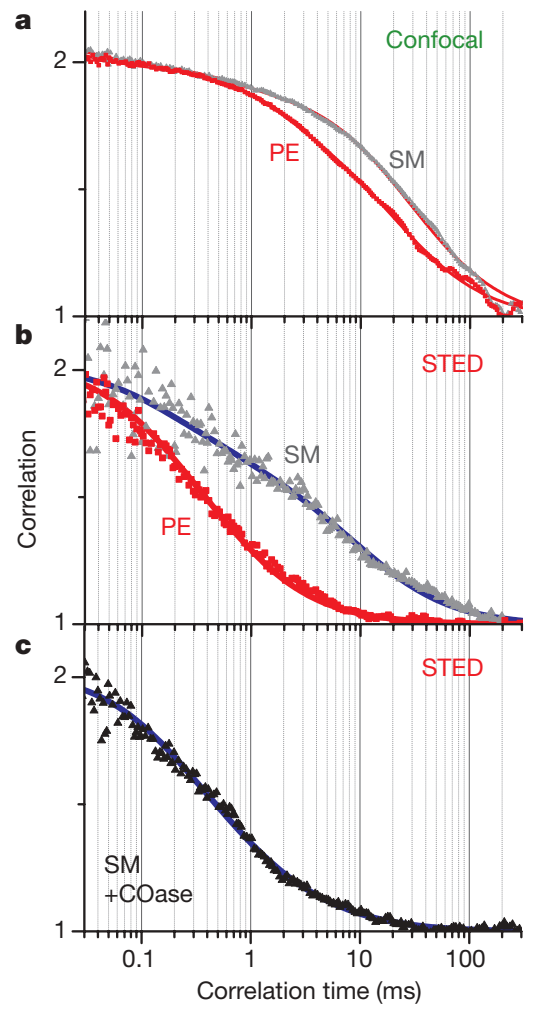

Figure 2 | FCS of Atto647N-labelled phosphoethanolamine and sphingomyelin plasma membrane diffusion. a-c, Normalized correlation data of phosphoethanolamine (PE, red dots) and sphingomyelin (SM, grey dots) for confocal (a) and STED (b) recording, and of sphingomyelin (c) after cholesterol depletion by the addition of cholesterol oxidase (COase, black dots) for STED recording. The STED but not the confocal data reveal cholesterol-assisted heterogeneous diffusion of sphingomyelin. Red lines in $\mathbf{a}$ and $\mathbf{b}$ denote single-species fit; $\tau_{D}=19$ (phosphoethanolamine, confocal), 28 (sphingomyelin, confocal) and $0.45 \mathrm{~ms}$ (phosphoethanolamine, STED). Blue lines denote fit assuming two dissimilar modalities of focal transits: $\tau_{D 1}=0.45 \mathrm{~ms}, \tau_{D 2}=10 \mathrm{~ms}$ and $A_{2}=64 \%$ (sphingomyelin, in b) and $\tau_{D 2}=4 \mathrm{~ms}$ and $A_{2}=15 \%$ (sphingomyelin + cholesterol oxidase, in c).

The hindered sphingolipid and GPI-anchor diffusion may be caused by various molecular interactions. However, we can exclude the floating of temporally stable lipid complexes or domains through the membrane, because this would manifest itself as a linear dependence of $\tau_{D 2}$ on the reduced focal area $d^{2}$ created by STED. Moreover, our data demonstrate that during the trapping, the sphingolipids or GPI-anchored molecules remain within a $<20-\mathrm{nm}$ diameter area. This conclusion is on the basis of the fact that for 30-60 nm large spots, the transit time $\tau_{D 2}$ and the fraction $A_{2}$ of diffusion-hindered events is constant (Supplementary Information). These findings are supported by alternative evaluations of the FCS data applying anomalous diffusion models (Supplementary Fig. 3), by the STED images recorded for sphingomyelin (Supplementary Fig. 10), and by Monte Carlo simulations of hindered lipid dynamics (Supplementary Fig. 9).

Altogether, the evolving picture of the observed sphingolipid and GPI-anchor dynamics is that of transient formations of cholesterolassisted molecular complexes, such as lipid-protein binding or lipid shells (compare Supplementary Information). Although an entire complex of for example several lipid and protein molecules may (temporarily) be of larger spatial scales, the trapped molecule dwells only within $<20$-nm diameter areas. The diffusion of the complexes must be slow, because they must diffuse through the focal spot within a time period that is longer than the trapping time, that is, with a diffusion constant $D \ll 0.2 \times d^{2} / \tau_{\text {trap }} \approx 10^{-10} \mathrm{~cm}^{2} \mathrm{~s}^{-1}$.

The linear dependence of the transit time of the freely diffusing lipids on the subdiffraction detection area (and their average particle number per area, Supplementary Fig. 11) proves that our data are not

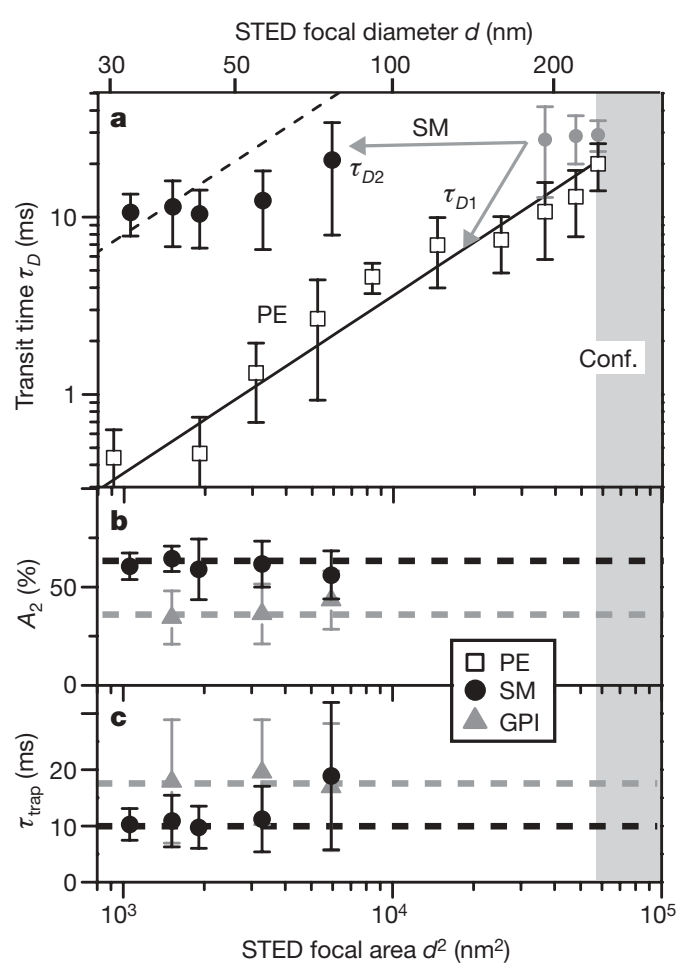

Figure 3 | Molecular transit through nanoscale areas in live cell plasma membrane. The parameters measured by STED-FCS in focal detection areas ranging from $d=250 \mathrm{~nm}$ (conf., confocal; grey area) down to $30 \mathrm{~nm}$ in diameter. a, The transit times $\tau_{D}$ determined for phosphoethanolamine (PE, open squares) decrease linearly with the area $d^{2}$, confirming free diffusion (solid line). Whereas large areas $>180 \mathrm{~nm}$ in diameter yield just a single transit time $\tau_{D}$ of sphingomyelin (SM, grey dots), detection areas $<80 \mathrm{~nm}$ in diameter reveal two distinct modalities of molecular transits (grey arrows), with $\tau_{D 1}=\tau_{D}$ (phosphoethanolamine) (solid line) and $\tau_{D 2}$ demonstrating hindered diffusion. The $\tau_{D 2}$ plateau found for small $d$ indicates transient trapping of sphingomyelin. Slowed-down free diffusion of sphingomyelin can be ruled out because such behaviour would display linear dependence of $\tau_{D}$ on $d^{2}$, as exemplified for $D=2 \times 10^{-10} \mathrm{~cm}^{2} \mathrm{~s}^{-1}$ (dashed line).

b, c, Fraction $A_{2}$ (a) and trapping time $\tau_{\text {trap }}$ (c) of transits of sphingomyelin (black dots) and GPI-anchor (grey triangles) exhibiting transient trapping. Standard deviations given as error bars result from averaging over more than thirty FCS measurements on different cells.

compromised by photobleaching, local heating or radical formation. Even if some molecules were bleached on passing the doughnut of STED light, the diffusion of those that have entered the detection area is less affected. In fact, the confocal measurements are more prone to bleaching artefacts due to the inherently longer transit time through the detection area. Thus the traces in Figs 1 and 2 show the ability of STED microscopy to provide a unique and hitherto unrecognized access to the dynamics of single molecules in nanosized areas in living cells, thereby greatly expanding the potential of the popular FCS technique. STED-FCS is complementary to single-molecule tracking because several molecules per subdiffraction area may contribute to the signal, allowing a fast gathering of statistically accurate data. Besides, the spot size of a STED microscope is physically predefined and the spot can be directed to arbitrary coordinates within a cell. All these features have provided a unique combination of temporal and spatial resolution required to quantify the nanoscale dynamics of the small lipid molecules in the cellular membrane. Thus the trapped molecular diffusion revealed in this work is only a prelude of a new class of nanoscale biomolecular studies that are to follow.

\section{METHODS SUMMARY}

In our STED microscope, the diffraction-limited focal spot of excitation light is overlapped with a doughnut-shaped spot of STED light that switches off the ability of the label to fluoresce, thus confining the origin of the fluorescence to 
the doughnut centre (Supplementary Fig. 1). With the intensity $I_{\mathrm{s}}$ being a characteristic of the fluorophore and the STED light, and $I \gg I_{\mathrm{s}}$ denoting the doughnut peak intensity, the diameter of the detection area can be tuned as $d \approx \lambda /\left(2 \mathrm{NA} \sqrt{1+I / I_{\mathrm{s}}}\right) \ll 250 \mathrm{~nm}$, with NA denoting the numerical aperture of the lens, and $\lambda$ the wavelength used for STED ${ }^{25,26}$. We used a $76-\mathrm{MHz}$ train of 250-300 ps pulses for STED at 770-780 nm, and an average focal power of up to $380 \mathrm{~mW}$. Imprinting a helical phase delay on the STED laser wavefront rendered a doughnut-shaped focal spot. The STED pulses triggered a 633-nm pulsed laser diode for fluorescence excitation with average powers of 5-8 $\mu \mathrm{W}$. Laser focusing and collection of the fluorescence was performed by an NA 1.42 oil immersion lens. The signal was detected by avalanche photo diodes together with a correlator or single-photon counting hardware. The experiments were run and analysed using Imspector as well as self-written software. The focal diameters $d$ of the STED microscope were calibrated by imaging 20-nm diameter fluorescent beads (Supplementary Fig. 1). Labelling of the lipids with the organic dye Atto647N was accomplished by chemical synthesis and of the GPI-anchor by acyl carrier protein (ACP)-tagging, respectively. Incubation with the fluorescent lipid-BSA complexes or GPI-ACP plasmid transfection resulted in molecular insertion into the plasma membrane of living PtK2 cells. Measurements were performed in cell culture medium at $27-37^{\circ} \mathrm{C}$, using a temperature-controlled sample chamber and objective lens heater, and by placing the foci of the inverted microscope randomly on the lower plasma membrane.

Full Methods and any associated references are available in the online version of the paper at www.nature.com/nature.

\section{Received 6 September; accepted 29 October 2008.}

\section{Published online 21 December 2008.}

1. Simons, K. \& Ikonen, E. Functional rafts in cell membranes. Nature 387, 569-572 (1997).

2. Brown, D. A. \& London, E. Structure and function of sphingolipid- and cholesterolrich membrane rafts. J. Biol. Chem. 275, 17221-17224 (2000).

3. Fielding, C. J. Lipid Rafts and Caveolae (Wiley-VCH, 2006).

4. Jacobson, K., Mouritsen, O. G. \& Anderson, G. W. Lipid rafts: at a crossroad between cell biology and physics. Nature Cell Biol. 9, 7-14 (2007).

5. Hanzal-Bayer, M. F. \& Hancock, J. F. Lipid rafts and membrane traffic. FEBS Lett. 581, 2098-2104 (2007).

6. Munro, S. Lipid rafts: elusive or illusive? Cell 115, 377-388 (2003).

7. Lommerse, P. H. M., Spaink, H. P. \& Schmidt, T. In vivo plasma membrane organization: results of biophysical approaches. Biochim. Biophys. Acta 1664, 119-131 (2004)

8. Hancock, J. F. Lipid rafts: contentious only from simplistic standpoints. Nature Rev. Mol. Cell Biol. 7, 456-462 (2006).

9. Shaw, A. S. Lipid rafts: now you see them, now you don't. Nature Immunol. 7, 1139-1142 (2006).

10. Hell, S. W. \& Wichmann, J. Breaking the diffraction resolution limit by stimulated emission: stimulated emission depletion microscopy. Opt. Lett. 19, 780-782 (1994).

11. Pike, L. J. Rafts defined: a report on the Keystone symposium on lipid rafts and cell function. J. Lipid Res. 47, 1597-1598 (2006).

12. Fujita, A. et al. Gangliosides GM1 and GM3 in the living cell membrane form clusters susceptible to cholesterol depletion and chilling. Mol. Biol. Cell 18, 2112-2122 (2007).
13. Binnig, G., Quate, C. F. \& Gerber, C. Atomic force microscope. Phys. Rev. Lett. 56, 930-933 (1986).

14. Pohl, D. W., Denk, W. \& Lanz, M. Optical stethoscopy: Image recording with resolution $\lambda / 20$. Appl. Phys. Lett. 44, 651-653 (1984).

15. Zacharias, D. A., Violin, J. D., Newton, A. C. \& Tsien, R. Y. Partitioning of lipidmodified monomeric GFPs into membrane microdomains of live cells. Science 296, 913-916 (2002).

16. Saxton, M. J. \& Jacobson, K. Single particle tracking: applications to membrane dynamics. Annu. Rev. Biophys. Biomol. Struct. 26, 373-399 (1997).

17. Schütz, G. J., Kada, G., Pastushenko, V. P. \& Schindler, H. Properties of lipid microdomains in a muscle cell membrane visualized by single molecule microscopy. EMBO J. 19, 892-901 (2000).

18. Fujiwara, T., Ritchie, K., Murakoshi, H., Jacobson, K. \& Kusumi, A. Phospholipids undergo hop diffusion in compartmentalized cell membrane. J. Cell Biol. 157, 1071-1081 (2002).

19. Yechiel, E. \& Edidin, M. Micrometer-scale domains in fibroblast plasma membranes. J. Cell Biol. 105, 755-760 (1987).

20. Feder, T. J., Brust-Mascher, I., Slattery, J. P., Baird, B. A. \& Webb, W. W. Constrainted diffusion or immobile fraction on cell surfaces: a new interpretation. Biophys. J. 70, 2767-2773 (1996).

21. Fahey, P. F. et al. Lateral diffusion in planar lipid bilayers. Science 195, 305-306 (1977).

22. Schwille, P., Korlach, J. \& Webb, W. W. Fluorescence correlation spectroscopy with single-molecule sensitivity on cell and model membranes. Cytometry 36, 176-182 (1999).

23. Wawrezinieck, L., Rigneault, H., Marguet, D. \& Lenne, P.-F. Fluorescence correlation spectroscopy: diffusion laws to probe the submicron cell membrane organization. Biophys. J. 89, 4029-4042 (2005).

24. Wenger, J. et al. Diffusion analysis within single nanometric apertures reveals the ultrafine cell membrane organization. Biophys. J. 92, 913-919 (2007).

25. Willig, K. I., Rizzoli, S. O., Westphal, V., Jahn, R. \& Hell, S. W. STED-microscopy reveals that synaptotagmin remains clustered after synaptic vesicle exocytosis. Nature 440, 935-939 (2006).

26. Hell, S. W. Far-field optical nanoscopy. Science 316, 1153-1158 (2007).

27. Magde, D., Elson, E. L. \& Webb, W. W. Thermodynamic fluctuations in a reacting system - measurement by fluorescence correlation spectroscopy. Phys. Rev. Lett. 29, 705-708 (1972).

Supplementary Information is linked to the online version of the paper at wWw.nature.com/nature.

Acknowledgements We thank J. Jethwa, B. Rankin and M. Hilbert for critical reading, K. Willig for help with the setup, T. Lang, R. Wagner and H. Rigneault for valuable discussions, R. Machinek and $\mathrm{H}$. Frauendorf for recording the NMR and mass spectra, and S. Yan for help with the synthesis.

Author Contributions C.R. and B.H. performed experiments, C.E. and C.R. analysed data, R.M. prepared samples and performed washing experiments, G.S., K.S., S.P. and V.N.B. synthesized fluorescently labelled lipids and performed

chromatography, C.v.M., A.S., C.R. and C.E. realized and analysed simulated data, C.E. and S.W.H. designed experiments and wrote the paper. All authors discussed the results and commented on the manuscript.

Author Information Reprints and permissions information is available at www.nature.com/reprints. Correspondence and requests for materials should be addressed to S.W.H. (shell@gwdg.de) or C.E. (ceggeli@gwdg.de). 


\section{METHODS}

Cell culture. The epithelial cell line PtK2 was grown as previously described ${ }^{28}$. The cells were seeded on number 1 thickness standard glass coverslips (diameter $25 \mathrm{~mm}$ ) to a confluence of about $80 \%$ and grown at $37^{\circ} \mathrm{C}$ in a water-saturated atmosphere of $5 \% \mathrm{CO}_{2}$ in air. All media and supplements were purchased from Invitrogen.

Atto647N-labelled lipids. We used the organic dye Atto647N (fluorescence excitation and emission maxima at 645 and $670 \mathrm{~nm}$, respectively; Atto-Tec) as a fluorescence marker. $N$-(Atto647N)-1,2-dipalmitoyl-sn-glycero-3-phosphoethanolamine (Atto647N-phosphoethanolamine, referred to as phosphoethanolamine) and $N$-(Atto647N)-sphingosylphosphocholine ( $N$-Atto647-sphingomyelin, Atto647N-sphingomyelin, referred to as sphingomyelin) were purchased from Atto-Tec. The Atto647N-labelled ganglioside GM1 was synthesized as outlined in the Supplementary Information starting from GM1 (ALEXIS Biochemicals). We performed a series of control experiments using lipids labelled at different positions either with lipophilic or hydrophilic fluorescent labels. The control experiments together with the structures of all lipids are depicted in Supplementary Figs 7 and 8 . The experiments indicate no observable influence of the used label on the dynamic characteristics of the labelled lipids.

Plasma membrane insertion of the fluorescent lipids. Complexes of the labelled lipids and bovine serum albumin (BSA) were prepared and incubated with the cells according to a slightly modified protocol than that previously described $^{29,30}$; as detailed in the Supplementary Information.

Glycosylphosphatidylinositol anchor. We used an ACP tag (Covalys Bioscience) for labelling of the GPI-anchor with the organic dye Atto647N. Twenty-four hours after transfection with the plasmid pAEMXT-ACPwt-GPI (Covalys), we incubated PtK2 cells expressing GPI-anchored ACP for 30 min at $37^{\circ} \mathrm{C}$ in complete medium supplemented with $5 \mu \mathrm{M} \mathrm{CoA}$-Atto647N (AttoTec; $\mathrm{CoA}$, coenzyme A), $5 \mathrm{mM} \mathrm{MgCl} 2$ (Fluka) and $1 \mu \mathrm{M} \mathrm{ACP}$ synthase (Covalys). Afterwards, the cells were washed three times and prepared for measurements using DMEM containing no phenol red. The cells were measured $24 \mathrm{~h}$ after transfection. Control experiments with different fluorescent markers precluded an observable influence of the label on GPI trapping (Supplementary Information).

Treatment with cholesterol oxidase and $\beta$-cyclodextrin. To modify the cholesterol contents of the plasma membrane, the cells were either treated with $1 \mathrm{U} \mathrm{ml}^{-1}$ Streptomyces sp. cholesterol oxidase (Sigma, stock solution $34 \mathrm{U} \mathrm{ml}^{-1}$ in $50 \mathrm{mM} \mathrm{KH}_{2} \mathrm{PO}_{4}, \mathrm{pH} 7.5$ ) in HDMEM (HEPES + DMEM) and then washed in HDMEM (see Supplementary Information), or treated with $10 \mathrm{mM} \beta$-cyclodextrin (Sigma) in HDMEM (without phenol red) for $30 \mathrm{~min}$ at $37^{\circ} \mathrm{C}$. The cholesterol oxidase or $\beta$-cyclodextrin treatment was performed either before or after the insertion of the fluorescent lipid analogues into the plasma membrane.

STED microscope. We implemented a confocalized setup in which the excitation of the dye was performed with a $633 \mathrm{~nm}$ pulsed laser diode $(\approx 80 \mathrm{ps}$ pulse width, LDH-P-635, Picoquant). The STED beam was provided by a titanium: sapphire laser system (MaiTai, Spectra-Physics) operating at 770-780 nm with a repetition rate of $76 \mathrm{MHz}$; this beam also provided the trigger for the excitation laser. The STED laser power was controlled and stabilized by a power controller unit (LPC, Brockton Electronics). After passing a $30-\mathrm{cm}$ optical glass rod, the linear-polarized light was coupled into a $120 \mathrm{~m}$ long polarization maintaining single-mode fibre (AMS Technologies) for stretching the pulses to 250-300 ps. The pulse timing of both lasers was adjusted using a home-built electronic delay unit. After spatial overlay of both laser beams with appropriate dichroic filters (AHF Analysentechnik) they were directed to a beam-scanning device (mirror tilting system PSH 10/2, Piezosystem Jena) and then directed into the microscope (DMIRBE, Leica Microsystems). We used an oil immersion objective (PLAPON $\times 60, \mathrm{NA}=1.42$, Olympus) to focus the laser light to a spot on the sample and to collect the fluorescence. The beam-scanning device allowed an exact control of the lateral position of the focal spots on the sample and enabled scanning. The axial position of the focal spots was adjusted by an objective lens positioning system (MIPOS 250, Piezosystem Jena). The doughnut-shaped focal spot of the STED beam featuring a central zero intensity was produced by introducing a phase-modifying plate (RPC Photonics) into the beam path, imprinting on the wave front a helical phase $\operatorname{ramp} \exp (\mathrm{i} \varphi)$ with $0 \leq \varphi \leq 2 \pi$. A $\lambda / 4$-plate ensured circular polarization of the STED and of the excitation beam $^{31,32}$. The fluorescence was imaged back over the beam-scanning device and coupled into a multi-mode fibre splitter (Fibre Optic Network Technology) with an aperture size corresponding to $\times 1.4$ the magnified excitation spot. The 50:50 split fluorescence signal was then detected by two singlephoton counting units (avalanche photo diode SPCM-AQR-13-FC, Perkin Elmer Optoelectronics). We blocked potential contributions from scattered laser light or unwanted autofluorescence by appropriate emission filters (AHF Analysentechnik). The fluorescence counts were further processed by a hardware correlator card (Flex02-01D, Correlator.com) for FCS measurements or by a single-photon counting PC card (SPC 830, Becker \& Hickl GmbH) for direct single-molecule analysis. The focal intensity distribution of the excitation and STED light were measured by scanning a scattering gold bead of sub-diffraction diameter ( $80 \mathrm{~nm}$ gold colloid, En.GC80, BBinternational) using a non-confocal detector (MP 963 Photon Counting Module, Perkin Elmer). The (pulse) intensity of the excitation light was $3-5 \mathrm{MW} \mathrm{cm}^{-2}\left(\sim 25 \mathrm{~kW} \mathrm{~cm}^{-2}\right.$ on average), stemming from $P=5-8 \mu \mathrm{W}$ on the confocal spot of diameter (full-width-at-half maximum) $d=250 \mathrm{~nm}$.

Measurements. We analysed the lipid dynamics by placing the foci on random positions in the lower plasma membrane facing the coverslip and completed all measurements before disruptive internalization, or any morphological changes in the cell could take place. The measurement times were kept short $(\sim 15 \mathrm{~s})$ to avoid biasing distortion of the correlation data due to very infrequent transits of bright particles such as cell debris. The coverslips were mounted in a special microscope chamber (RC-40, Warner Instruments). This chamber together with an objective heater (Bioptechs Inc.) allowed a precise control of the sample's temperature. We performed most measurements at $27-37^{\circ} \mathrm{C}$ in HDMEM as outlined in detail in the Supplementary Information. We excluded diffusion of non-integrated lipids (or dye tags) by control measurements in between the cells.

28. Osborn, M., Franke, W. W. \& Weber, K. Visualization of a system of filaments 7-10 nm thick in cultured cells of an epithelioid line (PtK2) by immunofluorescence microscopy. Proc. Natl Acad. Sci. USA 74, 2490-2494 (1977).

29. Martin, O. C.\& Pagano, R. C. Internalization and sorting of a fluorescent analogue of glucosylceramide to the Golgi apparatus of human skin fibroblasts: utilization of endocytic and nonendocytic transport mechanisms. J. Cell Biol. 125, 769-781 (1994).

30. Schwarzmann, G., Hofmann, P., Pütz, U. \& Albrecht, B. Demonstration of direct glycosylation of nondegradable glucosylceramide analogs in cultured cells. J. Biol. Chem. 270, 21271-21276 (1995).

31. Keller, J., Schönle, A. \& Hell, S. W. Efficient fluorescence inhibition patterns for RESOLFT microscopy. Opt. Express 15, 3361-3371 (2007).

32. Willig, K. I. et al. Nanoscale resolution in GFP-based microscopy. Nature Methods 3, 721-723 (2006). 